\title{
A POLYNOMIAL-TIME INTERIOR-POINT ALGORITHM FOR CONVEX QUADRATIC SEMIDEFINITE OPTIMIZATION*
}

\author{
Y.Q. BAI ${ }^{1}$, F.Y. WANG ${ }^{1}$ AND X.W. LUO $^{1}$
}

\begin{abstract}
In this paper we propose a primal-dual interior-point algorithm for convex quadratic semidefinite optimization problem. The search direction of algorithm is defined in terms of a matrix function and the iteration is generated by full-Newton step. Furthermore, we derive the iteration bound for the algorithm with small-update method, namely, $O\left(\sqrt{n} \log \frac{n}{\varepsilon}\right)$, which is best-known bound so far.
\end{abstract}

Keywords. Convex quadratic semidefinite optimization, interior-point algorithm, small-update method, iteration bound, polynomial-time.

Mathematics Subject Classification. 90C05, 90C51.

\section{INTRODUCTION}

Let $S^{n}, S_{+}^{n}, S_{++}^{n}$ denote the cone of symmetric, symmetric positive semidefinite and symmetric positive $n \times n$ matrices, respectively.

Received September 15, 2009. Accepted September 1, 2010.

* This research is supported by grants of National Natural Science Foundation of China (No. 11071158), and Shanghai Leading Academic Discipline Project (No. S30104).

1 Department of Mathematics, Shanghai University, Shanghai, 200444, P.R. China. yqbai@shu.edu.cn 
We consider the standard convex quadratic semidefinite optimization (CQSDO) problem as follows:

$$
\begin{array}{cl}
\min & C \bullet X+\frac{1}{2} X \bullet Q X \\
\text { s.t. } & A_{i} \bullet X=b_{i}, \quad i=1, \ldots, m, \\
& X \succeq 0,
\end{array}
$$

where $A_{i} \in S^{n}, i=1, \ldots, m, b=\left(b_{1}, b_{2}, \ldots, b_{m}\right)^{T} \in R^{m}, C \in S^{n}, Q \in S_{+}^{n}$. The lagrangian dual problem of $(P)$ is

$$
\begin{array}{ll}
\max & b^{T} y-\frac{1}{2} X \bullet Q X \\
\text { s.t. } & \sum_{i=1}^{m} y_{i} A_{i}-Q X+S=C, \quad i=1, \ldots, m, \\
& S \succeq 0 .
\end{array}
$$

The CQSDO problems first appeared in the research of Kojima et al. [11] and they realized that the CQSDO problems were a special case of monotone semidefinite linear complementarity problems (SDLCPs). In addition, if the matrices $C, A_{i}(i=1, \ldots, m)$ and $X$ are diagonal, $(P)$ reduces to a convex quadratic optimization problem. If $Q=0$, the CQSDO problem is just semidefinite optimization (SDO) problem. Moreover, some optimization problems can transform to the CQSDO problems, such as the nearest Euclidean distance matrix problem, the nearest correlation matrix problem, Toh et al., we refer the reader to [16] for details, and the CQSDO problems have some important implications in molecular conformation problems in chemistry and multidimensional scaling and multivariate analysis problems in statistics, Alfakih et al. We refer the reader to [3] wherein some other applications of CQSDO are cited. Furthermore, some efficient interior-point algorithms have been proposed to CQSDO, such as Nie and Yuan in $[13,14]$ proposed two algorithms for solving CQSDO problems, which are predictor-corrector algorithm and potential reduction algorithm, respectively, and Toh proposed an inexact primal-dual path-following algorithm for CQSDO in [15]. Wang and Bai [19] designed an interior point algorithm based on a parametric kernel function and get the large- and small-update iteration bound. Recently, Darvay [6] proposed a new technique for finding a class of search directions. Based on this technique, the author designed a new primal-dual interior-point algorithm for linear optimization (LO) with small-update method, and obtained iteration bound $O\left(\sqrt{n} \log \frac{n}{\varepsilon}\right)$. Achache [1] extended this technique to convex quadratic optimization. Later on, Wang and Bai [18] successfully extended this technique to SDO and obtained the same iteration bound as LO.

Motivated by their work, we propose a new primal-dual path-following interior point algorithm for CQSDO. We use a new method to find the search directions and analyze the algorithm. Moreover, we derive the iteration bound for the algorithm 
with small-update method is $O\left(\sqrt{n} \log \frac{n}{\varepsilon}\right)$, which is the same as LO and SDO and is the best known bound so far.

The paper is organized as follows: in Section 1, we recall briefly basic results on matrices and matrix functions and explain the concepts of the central path. In Section 2, we use the new technique to find the search directions. In Section 3, we present the generic primal-dual interior-point algorithm for CQSDO. In Section 4, we derive the algorithm is well defined and can solve the CQSDO problem in polynomial time. Finally, some conclusions are given in Section 5.

Some notations used throughout the paper are as follows: $\|\cdot\|_{F}$ and $\|\cdot\|_{2}$ denote the Frobenius norm and the spectral norm for matrices, respectively. $E$ denotes the identity matrix. $A \succeq B$ (or $A \succ B$ ) means $A-B$ is positive semidefinite (or positive definite). We denote $A \bullet B=\operatorname{Tr}\left(\mathrm{A}^{\mathrm{T}} \mathrm{B}\right)$ i.e., the trace of $A^{T} B$. For any $Q \in S_{++}^{n}$, the $Q^{1 / 2}$ (or $\sqrt{Q}$ ) denotes its symmetric square root. When $\lambda$ is a vector, we use the diagonal matrix $\Lambda$ with entries $\lambda_{i}$ by $\operatorname{diag}(\lambda)$. We assume that the eigenvalues of positive semi-definite $V$ are listed according to the order of their values such that $\lambda_{1}(V) \geq \lambda_{2}(V) \geq \cdots \geq \lambda_{n}(V)$. The notation $g(x)=O(x)$ means that $g(x) \leq \bar{c} x$ for some positive constant $\bar{c}$.

\section{Preliminaries}

\subsection{MATRICES AND MATRIX FUNCTION}

We first recall some knowledge of matrices and matrix functions, which we can refer to $[7,8]$ for the details.

Definition 1.1. Let $A \in \mathcal{R}^{n \times n}$, then the trace of $A$ is the sum of the diagonal elements of matrix $A$, we denoted it as $\operatorname{Tr}(\mathrm{A})$.

Lemma 1.2. Let $A, B \in \mathcal{R}^{n \times n}$, then

(I) $\operatorname{Tr}(\mathrm{A})=\sum_{\mathrm{i}=1}^{\mathrm{n}} \lambda_{\mathrm{i}}(\mathrm{A})$, where $\lambda_{i}(A)$ is the $i$ th eigenvalue of matrix $A$.

(II) $\operatorname{Tr}(\mathrm{A})=\operatorname{Tr}\left(\mathrm{A}^{\mathrm{T}}\right)$.

(III) $\operatorname{Tr}(\mathrm{AB})=\operatorname{Tr}(\mathrm{BA})$.

(IV) $\operatorname{Tr}(\mathrm{A}+\mathrm{B})=\operatorname{Tr}(\mathrm{A})+\operatorname{Tr}(\mathrm{B})$. Specially, if $B$ is a skew-symmetric matrix, then $\operatorname{Tr}(\mathrm{A}+\mathrm{B})=\operatorname{Tr}(\mathrm{A})$.

Definition 1.3. Let $A \in \mathcal{R}^{n \times n}$, the Frobenius norm and spectral norm are respectively defined as

$$
\|A\|_{F}=\sqrt{\operatorname{Tr}\left(\mathrm{A}^{\mathrm{T}} \mathrm{A}\right)}=\sqrt{\sum_{i=1}^{n} \sum_{i=1}^{n} a_{i j}}\left(=\sqrt{\sum_{i=1}^{n} \lambda_{i}^{2}(A)} \text { if } A \in S_{+}^{n}\right)
$$

and

$$
\|A\|_{2}=\sqrt{\lambda_{\max }\left(A^{T} A\right)}\left(=\lambda_{\max }(A) \text { if } A \in S_{+}^{n}\right) .
$$


Theorem 1.4 (Spectral theorem for symmetric matrices in [21]). The real $n \times n$ matrix $A$ is symmetric if and only if there exists a matrix $Q \in \mathcal{R}^{n \times n}$ such that $Q^{T} Q=E$ and $Q^{T} A Q=\Lambda$ where $\Lambda=\operatorname{diag}\left(\lambda_{1}, \lambda_{2}, \ldots, \lambda_{\mathrm{n}}\right)$.

From Theorem 1.4, we can easily get the following corollary.

Corollary 1.5. Let $A \in S^{n}$, then we have $A^{2} \in S^{n}$ and $\lambda_{i}\left(A^{2}\right)=\lambda_{i}^{2}(A)$.

Now, we are ready to give the matrix functions. Suppose that $\psi(t)$ is a real valued function and its domain is $[0,+\infty)$, moreover, the derivative of $\psi(t)$ exists and $\psi^{\prime}(t)>0$ for $t>0$.

Definition 1.6. Let $V \in S_{+}^{n}$ and

$$
V=Q^{T} \operatorname{diag}\left(\lambda_{1}(\mathrm{~V}), \lambda_{2}(\mathrm{~V}), \ldots, \lambda_{\mathrm{n}}(\mathrm{V})\right) \mathrm{Q}
$$

where $Q$ is any orthonormal matrix that diagonalizes $V$. Then the (matrix valued) matrix function $\psi(V): S_{+}^{n} \mapsto S^{n}$ is defined as

$$
\psi(V)=Q^{T} \operatorname{diag}\left(\psi\left(\lambda_{1}(\mathrm{~V})\right), \psi\left(\lambda_{2}(\mathrm{~V})\right), \ldots, \psi\left(\lambda_{\mathrm{n}}(\mathrm{V})\right) \mathrm{Q}\right.
$$

Furthermore, we define the derivative of $\psi(V)$, denoted as $\psi^{\prime}(V)$. The $\psi^{\prime}(V)$ is given as follows:

$$
\psi^{\prime}(V)=Q^{T} \operatorname{diag}\left(\psi^{\prime}\left(\lambda_{1}(\mathrm{~V})\right), \psi^{\prime}\left(\lambda_{2}(\mathrm{~V})\right), \ldots, \psi^{\prime}\left(\lambda_{\mathrm{n}}(\mathrm{V})\right) \mathrm{Q}\right.
$$

Definition 1.7 (Def. 1.3.1 in [8]). Let $A, B \in R^{n \times n}, A$ and $B$ are called similar, denoted as $A \sim B$, if there exits an invertible matrix $P$, such that $A=P B P^{-1}$.

Definition 1.8. Let $A, B \in S^{n}$, the matrix $A$ and $B$ are approximately equivalent, if $A B=B A$ and the absolute value of the difference of their each corresponding eigenvalue is small enough. We denote it as $A \approx B$.

It is easily to get the following lemma by matrix knowledge.

Lemma 1.9. Let $A, B \in S^{n}$. If $A+B=E$, then $A B=B A$.

Lemma 1.10 (Lem. 2.5 in [18]). Let $A, B \in S^{n}$ and $A B=B A$, then

$$
\lambda_{i}(A+B)=\lambda_{i}(A)+\lambda_{i}(B), \quad i=1,2, \ldots, n .
$$

Furthermore, if $\left|\lambda_{i}(B)\right|$ is small enough, we have

$$
\psi(A+B) \approx \psi(A)+\psi(B) .
$$

\subsection{The CEntral PATH}

First, we assume that $(P)$ and its dual $(D)$ satisfy the interior-point condition (IPC), i.e., there exists $X \in \mathcal{P}, S \in \mathcal{D}$ with $X \succ 0, S \succ 0$, where $\mathcal{P}$ and $\mathcal{D}$ denote the feasible set of problem $(P)$ and its dual $(D)$, respectively. Under the 
assumption of IPC, the optimality condition for $(P)$ and $(D)$ can be written as follows:

$$
\begin{aligned}
A_{i} \bullet X & =b_{i}, \quad i=1, \ldots, m, X \succ 0, \\
\sum_{i=1}^{m} y_{i} A_{i}-Q X+S & =C, S \succ 0, \\
X S & =0 .
\end{aligned}
$$

We modify the above system by relaxing the third equation as follows:

$$
\begin{aligned}
A_{i} \bullet X & =b_{i}, \quad i=1, \ldots, m, X \succ 0, \\
\sum_{i=1}^{m} y_{i} A_{i}-Q X+S & =C, S \succ 0, \\
X S & =\mu E
\end{aligned}
$$

with $\mu>0$. Under the assumption the $(P)$ and $(D)$ satisfy the IPC, the system (4) has a unique solution, denoted by $(X(\mu), y(\mu), S(\mu))$. We call $X(\mu)$ the $\mu$-center of $(P)$ and $(y(\mu), S(\mu))$ the $\mu$-center of $(D)$. The set of $\mu$-centers (with $\mu$ running through positive real numbers) gives a homotopy path, which is called the central path of $(P)$ and $(D)$. If $\mu \rightarrow 0$, then the limit of the central path exists and since the limit points satisfy the complementarity condition, the limit yields optimal solutions for $(P)$ and $(D)[21]$.

\section{THE NEW SEARCH DIRECTIONS}

We use a new technique to find the search direction through replacing the third equation of the system (4) by $\psi\left(\frac{X S}{\mu}\right)=\psi(E)$, then the system (4) can be rewritten as

$$
\begin{aligned}
A_{i} \bullet X & =b_{i}, \quad i=1, \ldots, m, \quad X \succ 0, \\
\sum_{i=1}^{m} y_{i} A_{i}-Q X+S & =C, \quad S \succ 0, \\
\psi\left(\frac{X S}{\mu}\right) & =\psi(E) .
\end{aligned}
$$

For a given feasible iterate $(X, y, S)$ with $X, S \succ 0$, the search directions $\Delta X, \Delta y$, $\Delta S$ at the current iteration is the unique solution of the following Newton system

$$
\begin{aligned}
A_{i} \bullet(X+\Delta X) & =b_{i}, \quad i=1, \ldots, m, \\
\sum_{i=1}^{m}\left(y_{i}+\Delta y_{i}\right) A_{i}-Q(X+\Delta X)+(S+\Delta S) & =0, \\
\psi\left(\frac{(X+\Delta X)(S+\Delta S)}{\mu}\right) & =\psi(E) .
\end{aligned}
$$


Applying Lemma 1.10 and neglecting the term $\Delta X \Delta S$, then the third equation of (6) can be written as

$$
\psi\left(\frac{X S}{\mu}\right)+\psi^{\prime}\left(\frac{X S}{\mu}\right) \frac{X \Delta S}{\mu}+\psi^{\prime}\left(\frac{X S}{\mu}\right) \frac{S \Delta X}{\mu}=\psi(E) .
$$

Using the first two equations of the system (5) and the above equation, then system (6) can be written as

$$
\begin{aligned}
A_{i} \bullet \Delta X & =b_{i}, \quad i=1, \ldots, m \\
\sum_{i=1}^{m} \Delta y_{i} A_{i}-Q \Delta X+\Delta S & =0 \\
\Delta X+X \Delta S S^{-1} & =\mu\left(\psi^{\prime}\left(\frac{X S}{\mu}\right)\right)^{-1}\left(\psi(E)-\psi\left(\frac{X S}{\mu}\right)\right) S^{-1} .
\end{aligned}
$$

To keep $\Delta X$ and $\Delta S$ are symmetric, we use NT-symmetrization scheme in [12]. Now, we define

$$
P:=X^{1 / 2}\left(X^{1 / 2} S X^{1 / 2}\right)^{-1 / 2} X^{1 / 2}=S^{-1 / 2}\left(S^{1 / 2} X S^{1 / 2}\right)^{1 / 2} S^{-1 / 2} .
$$

We replace the term $X \Delta S S^{-1}$ in the third equation of the system (7) by $P \Delta S P^{T}$. Then we have

$$
\begin{aligned}
A_{i} \bullet \Delta X & =b_{i}, \quad i=1, \ldots, m \\
\sum_{i=1}^{m} \Delta y_{i} A_{i}-Q \Delta X+\Delta S & =0 \\
\Delta X+P \Delta S P^{T} & =\mu\left(\psi^{\prime}\left(\frac{X S}{\mu}\right)\right)^{-1}\left(\psi(E)-\psi\left(\frac{X S}{\mu}\right)\right) S^{-1} .
\end{aligned}
$$

Furthermore, let $D=P^{1 / 2}, V:=\frac{1}{\sqrt{\mu}} D^{-1} X D^{-1}=\frac{1}{\sqrt{\mu}} D S D$. Then we have

$$
V^{2}=\left(\frac{1}{\sqrt{\mu}} D^{-1} X D^{-1}\right)(\sqrt{\mu} D S D)=D^{-1} \frac{X S}{\mu} D .
$$

Using Definition 2.6, we have

$$
\psi\left(\frac{X S}{\mu}\right)=D \psi\left(V^{2}\right) D^{-1} \text { and } \psi^{\prime}\left(\frac{\mathrm{XS}}{\mu}\right)=\mathrm{D} \psi^{\prime}\left(\mathrm{V}^{2}\right) \mathrm{D}^{-1} .
$$

Furthermore, let

$$
\bar{A}_{i}:=\frac{1}{\sqrt{\mu}} D A_{i} D ; D_{X}:=\frac{1}{\sqrt{\mu}} D^{-1} \Delta X D^{-1} ; D_{S}:=\frac{1}{\sqrt{\mu}} D \Delta S D .
$$


Then (8) can be rewritten as

$$
\begin{aligned}
\bar{A}_{i} \bullet D_{X} & =0, \quad i=1, \ldots, m, \\
\sum_{i=1}^{m} \Delta y_{i} \bar{A}_{i}-\bar{Q} D_{X}+D_{S} & =0, \\
D_{X}+D_{S} & =P_{V},
\end{aligned}
$$

where $\bar{Q} D_{X}=D Q D D_{X} D^{2}$ and

$$
P_{V}=\sqrt{\mu} D^{-1}\left(D \psi^{\prime}\left(V^{2}\right) D^{-1}\right)^{-1}\left(\psi(E)-D \psi\left(V^{2}\right) D^{-1}\right) S^{-1} D^{-1} .
$$

In this paper, we use $\psi(t)=\sqrt{t}$, then

$$
P_{V}=2(E-V)
$$

We also have

$$
V^{2}+V P_{V}=V^{2}+2 V(E-V)=E-(E-V)^{2}=E-\frac{P_{V}^{2}}{4}
$$

For the further analysis of the algorithm, we define a norm-based proximity measure $\delta(X, S, \mu)$ as follows

$$
\delta(V):=\delta(X, S, \mu):=\frac{\left\|P_{V}\right\|_{F}}{2}=\|E-V\|_{F} .
$$

Through the first two equations of the system (11), we have

$$
D_{X} \bullet D_{S} \geq 0
$$

It is the largest difference between the CQSDO and the SDO, as for SDO case, $D_{X} \bullet D_{S}=0[18]$.

We can easily obtain

$$
\delta(V)=0 \Leftrightarrow V=E \Leftrightarrow D_{X}=D_{S}=0 \Leftrightarrow X S=\mu E .
$$

So the value of $\delta(V)$ is considered as a measure for the distance between the given pair $(X, y, S)$ and $\mu$-center $(X(\mu), y(\mu), S(\mu))$.

As we use $\psi(t)=\sqrt{t}$, we replace the $P_{V}$ by $2(E-V)$ in (11). Then solving (11), we have the new search directions $D_{X}$ and $D_{S}$, by using (10), we can get $\Delta X$ and $\Delta S$. If $(X, y, S) \neq(X(\mu), y(\mu), S(\mu))$, then $(\Delta X, \Delta y, \Delta S) \neq 0$. So we obtain the new full-Newton triple by

$$
\left(X_{+}, y_{+}, S_{+}\right)=(X, y, S)+(\Delta X, \Delta y, \Delta S)
$$




\section{The GENERIC INTERIOR-POINT ALGORITHM}

In this section, we propose the generic primal-dual interior-point algorithm for CQSDO as the following Figure 1.

\section{Primal-Dual Interior-Point Algorithm for CQSDO}

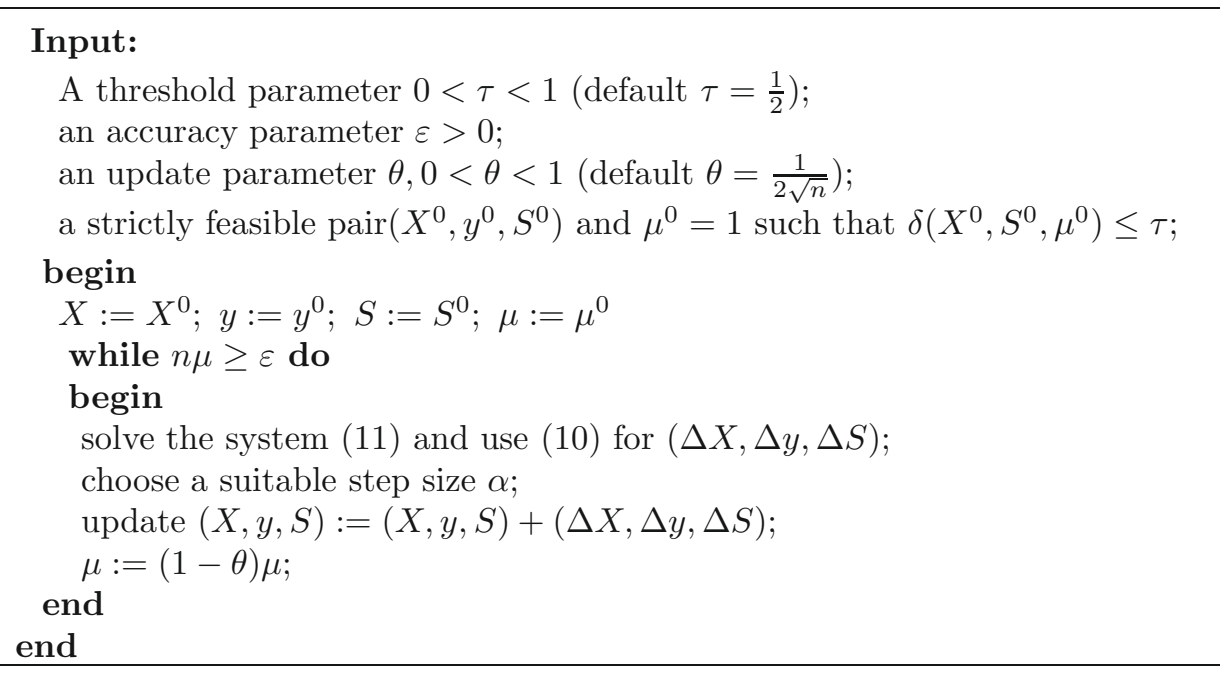

Figure 1. Primal - dual Interior Point Algorithm for CQSDO.

\section{Analysis of the Algorithm}

In this section, we will prove the algorithm is well defined and the CQSDO problem can be solved by this algorithm in polynomial time. In addition, we also prove the local quadratic convergence of the algorithm.

To simplify the discussion, let

$$
Q_{V}=D_{X}-D_{S}
$$

From the third equation of (11) and using (18), we get

$$
D_{X}=\frac{P_{V}+Q_{V}}{2}, \quad D_{S}=\frac{P_{V}-Q_{V}}{2}, \quad D_{X} D_{S}+D_{S} D_{X}=\frac{P_{V}^{2}-Q_{V}^{2}}{2} \text {. }
$$

From (14) and (15), we can derive

$$
\left\|Q_{V}\right\|_{F} \leq\left\|P_{V}\right\|_{F}=2 \delta(V) .
$$

Lemma 4.1 (Lem. 2.6 in [18]). Let $t>0$ and $V \in S_{+}^{n}$, then

$$
\left\|\left(t E-V^{2}\right)(t E+V)^{-1}\right\|_{F} \leq \frac{1}{t+\lambda_{\min }(V)}\left\|t E-V^{2}\right\|_{F} .
$$


Let $0 \leq \alpha \leq 1$, we define

$$
X(\alpha)=X+\alpha \Delta X, \quad S(\alpha)=S+\alpha \Delta S .
$$

Lemma 4.2 (Lem. 6.1 in [9]). Suppose that $X \succ 0$ and $S \succ 0$. If one has

$$
\operatorname{det}(\mathrm{X}(\alpha) \mathrm{S}(\alpha))>0, \quad \forall 0 \leq \alpha \leq \bar{\alpha}
$$

then $X(\bar{\alpha}) \succ 0$ and $S(\bar{\alpha}) \succ 0$.

Lemma 4.3 (Lem. 6.3 in [9]). Suppose that $Q \in S_{++}^{n}$ and $M \in \mathcal{R}^{n \times n}$ are skewsymmetric, i.e., $M=-M^{T}$. One has $\operatorname{det}(\mathrm{Q}+\mathrm{M})>0$. Moreover, if $\lambda_{i}(Q+M) \in$ $\mathcal{R}, i=1,2, \ldots, n$, then

$$
0<\lambda_{\min }(Q) \leq \lambda_{\min }(Q+M) \leq \lambda_{\max }(Q+M) \leq \lambda_{\max }(Q)
$$

which implies $Q+M \succ 0$.

The following lemma is very crucial to our analysis, under the condition we given, it also shows that the strict feasibility of the full Newton-step.

Lemma 4.4. Suppose $\delta:=\delta(X, S, \mu)<1$, then the full Newton step is strictly feasible.

Proof. From (21) and (10), we have

$$
\begin{aligned}
X(\alpha) S(\alpha) & =X S+\alpha(\Delta X S+X \Delta S)+\alpha^{2} \Delta X \Delta S \\
& =\mu D\left(V^{2}+\alpha\left(D_{X} V+V D_{S}\right)+\alpha^{2} D_{X} D_{S}\right) D^{-1} \\
& \sim \mu\left(V^{2}+\alpha\left(D_{X} V+V D_{S}\right)+\alpha^{2} D_{X} D_{S}\right)=Q(\alpha)+M(\alpha),
\end{aligned}
$$

where

$$
Q(\alpha)=\mu\left(V^{2}+\frac{1}{2} \alpha\left(D_{X} V+V D_{S}+V D_{X}+D_{S} V\right)+\frac{1}{2} \alpha^{2}\left(D_{X} D_{S}+D_{S} D_{X}\right)\right)
$$

and

$$
M(\alpha)=\mu\left(\frac{1}{2} \alpha\left(D_{X} V+V D_{S}-V D_{X}-D_{S} V\right)+\frac{1}{2} \alpha^{2}\left(D_{X} D_{S}-D_{S} D_{X}\right)\right) .
$$

It is clear to see that $M(\alpha)$ is skew-symmetric. From Lemma 4.3, if $Q(\alpha) \succ$ 0 , then $\operatorname{det}(\mathrm{Q}(\alpha)+\mathrm{M}(\alpha))>0$. As $X(\alpha) S(\alpha) \sim Q(\alpha)+M(\alpha)$, we can get $\operatorname{det}(\mathrm{X}(\alpha) \mathrm{S}(\alpha))>0$. Next, we just need to proof $Q(\alpha) \succ 0$. Applying (12), (13) 
and (19), we have

$$
\begin{aligned}
Q(\alpha) & =\mu\left(V^{2}+\frac{1}{2} \alpha\left(V P_{V}+P_{V} V\right)+\alpha^{2} \frac{P_{V}^{2}-Q_{V}^{2}}{4}\right) \\
& =\mu\left(V^{2}+\alpha V P_{V}+\alpha^{2} \frac{P_{V}^{2}-Q_{V}^{2}}{4}\right) \\
& =\mu\left((1-\alpha) V^{2}+\alpha\left(V^{2}+V P_{V}\right)+\alpha^{2} \frac{P_{V}^{2}-Q_{V}^{2}}{4}\right) \\
& =\mu\left((1-\alpha) V^{2}+\alpha\left(E-\frac{P_{V}^{2}}{4}\right)+\alpha^{2} \frac{P_{V}^{2}-Q_{V}^{2}}{4}\right) \\
& =\mu\left((1-\alpha) V^{2}+\alpha\left(E-(1-\alpha) \frac{P_{V}^{2}}{4}-\alpha \frac{Q_{V}^{2}}{4}\right)\right)
\end{aligned}
$$

Since $0 \leq \alpha \leq 1$ and (20), we have

$$
\begin{aligned}
\left.\|(1-\alpha) \frac{P_{V}^{2}}{4}+\alpha \frac{Q_{V}^{2}}{4}\right) \|_{F} & \leq(1-\alpha)\left\|\frac{P_{V}^{2}}{4}\right\|_{F}+\alpha\left\|\frac{Q_{V}^{2}}{4}\right\|_{F} \\
& \leq(1-\alpha) \frac{\left\|P_{V}\right\|_{F}^{2}}{4}+\alpha \frac{\left\|Q_{V}\right\|_{F}^{2}}{4} \\
& \leq(1-\alpha) \delta^{2}+\alpha \delta^{2}=\delta^{2}<1 .
\end{aligned}
$$

So we can get

$$
\left((1-\alpha) V^{2}+\alpha\left(E-(1-\alpha) \frac{P_{V}^{2}}{4}-\alpha \frac{Q_{V}^{2}}{4}\right)\right) \succ 0
$$

that is, $Q(\alpha) \succ 0$. Thus $\operatorname{det}(\mathrm{X}(\alpha) \mathrm{S}(\alpha))>0$. Since $X(0)=X \succ 0$ and $S(0)=$ $S \succ 0$, by Lemma 4.2, we have $X(1) \succ 0$ and $S(1) \succ 0$ for $\bar{\alpha}=1$. This completes the proof of the lemma.

Now we give an important lemma of local quadratic convergence of full Newtonstep algorithm.

Lemma 4.5. Suppose that $\delta=\delta(X, S, \mu)<1$, then we have

$$
\delta\left(X_{+}, S_{+}, \mu\right) \leq \frac{\delta^{2}}{1+\sqrt{1-\delta^{2}}} \leq \delta^{2}
$$

That is to say the full Newton-step algorithm satisfies local quadratic convergence.

Proof. Using the proof of Lemma 4.4 and (22) and letting $\alpha=1$, we have

$$
\frac{X_{+} S_{+}}{\mu} \sim E-\frac{Q_{V}^{2}}{4}+M,
$$


where

$$
M=\frac{1}{2}\left(D_{X} V+V D_{S}-V D_{X}-D_{S} V+D_{X} D_{S}-D_{S} D_{X}\right)
$$

Obviously, $M$ is a skew-symmetric matrix and $E-\frac{Q_{V}^{2}}{4} \succ 0$. So we derive that

$$
V_{+}^{2} \sim \frac{X_{+} S_{+}}{\mu} \sim E-\frac{Q_{V}^{2}}{4}+M
$$

By using Lemma 4.3, we have

$$
\lambda_{\min }\left(V_{+}^{2}\right)=\lambda_{\min }\left(E-\frac{Q_{V}^{2}}{4}+M\right) \geq \lambda_{\min }\left(E-\frac{Q_{V}^{2}}{4}\right) .
$$

Applying (20), we have

$$
\lambda_{\min }\left(V_{+}^{2}\right) \geq \lambda_{\min }\left(E-\frac{Q_{V}^{2}}{4}\right) \geq 1-\lambda_{\max }\left(\frac{Q_{V}^{2}}{4}\right) \geq 1-\left\|\frac{Q_{V}^{2}}{4}\right\| \geq 1-\frac{\left\|Q_{V}\right\|^{2}}{4} \geq 1-\delta^{2} .
$$

Therefore

$$
\lambda_{\min }\left(V_{+}\right) \geq \sqrt{1-\delta^{2}}
$$

By using Lemma 4.1 with $t=1$ and (23), we derive

$$
\begin{aligned}
\delta\left(X_{+}, S_{+}, \mu\right) & =\left\|E-V_{+}\right\|_{F}=\left\|\left(E-V_{+}\right)\left(E+V_{+}\right)\left(E+V_{+}\right)^{-1}\right\|_{F} \\
& =\left\|\left(E-V_{+}^{2}\right)\left(E+V_{+}\right)^{-1}\right\|_{F} \leq \frac{1}{1+\lambda_{\min }\left(V_{+}\right)}\left\|E-V_{+}^{2}\right\|_{F} \\
& =\frac{1}{1+\lambda_{\min }\left(V_{+}\right)} \sqrt{\operatorname{Tr}\left(\frac{\mathrm{Q}_{\mathrm{V}}^{2}}{4}-\mathrm{M}\right)^{2}} .
\end{aligned}
$$

Since $M$ is a skew-symmetric matrix, using (25) and Lemma 2.2, we get

$$
\begin{aligned}
\delta\left(X_{+}, S_{+}, \mu\right) & \leq \frac{1}{1+\lambda_{\min }\left(V_{+}\right)} \operatorname{Tr}\left(\frac{\mathrm{Q}_{\mathrm{V}}^{2}}{4}\right) \leq \frac{1}{1+\sqrt{1-\delta^{2}}}\left\|\frac{\mathrm{Q}_{\mathrm{V}}^{2}}{4}\right\|_{\mathrm{F}} \\
& \leq \frac{1}{1+\sqrt{1-\delta^{2}}} \frac{\left\|Q_{V}\right\|_{F}^{2}}{4} \leq \frac{\delta^{2}}{1+\sqrt{1-\delta^{2}}} \leq \delta^{2} .
\end{aligned}
$$

This completes the proof of the lemma.

Now, we give a critical lemma of computing the iteration bound, the proof of the lemma is similar to Lemma 6.5 in [18], so we just give the lemma without proof. 
Lemma 4.6. After a full-Newton step, then

$$
X_{+} \bullet S_{+} \leq n \mu .
$$

The following lemma gives a simple relation between $\delta$ and $\delta_{+}$after updating the $\mu$ to $(1-\theta) \mu$.

Lemma 4.7. Let $\delta<1$ and $\mu+=(1-\theta) \mu$, where $0<\theta<1$. Then

$$
\delta_{+}:=\delta\left(X_{+}, S_{+}, \mu_{+}\right) \leq \frac{\theta \sqrt{n}+\delta^{2}}{1-\theta+\sqrt{(1-\theta)\left(1-\delta^{2}\right)}} .
$$

Furthermore, if $\delta \leq \frac{1}{2}, \theta=\frac{1}{2 \sqrt{n}}$ and $n \geq 4$, then we have

$$
\delta\left(X_{+}, S_{+}, \mu_{+}\right) \leq \frac{1}{2}
$$

Proof. Using (23), (25) and applying Lemma 4.1 with $t=\sqrt{1-\theta}$, we derive

$$
\begin{aligned}
\delta\left(X_{+}, S_{+}, \mu_{+}\right) & =\left\|E-\sqrt{\frac{X_{+} S_{+}}{\mu_{+}}}\right\|_{F}=\frac{1}{\sqrt{1-\theta}}\left\|\sqrt{1-\theta} E-V_{+}\right\|_{F} \\
& =\frac{1}{\sqrt{1-\theta}}\left\|\left(\sqrt{1-\theta} E-V_{+}\right)\left(\sqrt{1-\theta} E+V_{+}\right)\left(\sqrt{1-\theta} E+V_{+}\right)^{-1}\right\|_{F} \\
& \leq \frac{1}{\sqrt{1-\theta}\left(\sqrt{1-\theta}+\lambda_{\min }\left(V_{+}\right)\right)}\left\|(1-\theta) E-V_{+}^{2}\right\|_{F} \\
& =\frac{1}{\sqrt{1-\theta}\left(\sqrt{1-\theta}+\lambda_{\min }\left(V_{+}\right)\right)} \sqrt{\operatorname{Tr}\left(-\theta \mathrm{E}+\frac{\mathrm{Q}_{\mathrm{V}}^{2}}{4}-\mathrm{M}\right)^{2}} .
\end{aligned}
$$

By $M$ is a skew-symmetric matrix and using (25), we have

$$
\begin{aligned}
\delta\left(X_{+}, S_{+}, \mu_{+}\right) & \leq \frac{1}{1-\theta+\sqrt{(1-\theta)\left(1-\delta^{2}\right)}}\left\|-\theta E+\frac{Q_{V}^{2}}{4}\right\|_{F} \\
& \leq \frac{1}{1-\theta+\sqrt{(1-\theta)\left(1-\delta^{2}\right)}}\left(\theta \sqrt{n}+\frac{\left\|Q_{V}\right\|_{F}^{2}}{4}\right) \\
& \leq \frac{1}{1-\theta+\sqrt{(1-\theta)\left(1-\delta^{2}\right)}}\left(\theta \sqrt{n}+\delta^{2}\right) .
\end{aligned}
$$

Furthermore, since $n \geq 4$ and $\delta \leq \frac{1}{2}$, we have

$$
1-\theta=1-\frac{1}{2 \sqrt{n}} \geq \frac{3}{4}
$$

and $\delta\left(X_{+}, S_{+}, \mu_{+}\right) \leq \frac{1}{2}$. 
From above lemmas and the default $\theta=\frac{1}{2 \sqrt{n}}$, we have that the $(X, S) \succ 0$ and $\delta<\frac{1}{2}$ are maintained during the algorithm. Hence, the algorithm is well defined.

Lemma 4.8. Suppose that $X^{0}$ and $S^{0}$ are strictly feasible, $\mu^{0}=\frac{X^{0} \bullet S^{0}}{n}$ and $\delta\left(X^{0}, S^{0}, \mu^{0}\right) \leq \frac{1}{2}$. Moreover, let $X^{k}$ and $S^{k}$ be the matrices obtained after $k$ iterations. Then the inequality $X^{k} \bullet S^{k} \leq \varepsilon$ is satisfied

$$
k \geq \frac{1}{\theta} \log \frac{X^{0} \bullet S^{0}}{\varepsilon} .
$$

Proof. From Lemma 4.6, we have

$$
X^{k} \bullet S^{k} \leq n \mu^{k}=n(1-\theta)^{k} \mu^{0}=(1-\theta)^{k} X^{0} \bullet S^{0}
$$

So, if we keep the inequality $X^{k} \bullet S^{k} \leq \varepsilon$ hold, we just need

$$
(1-\theta)^{k} X^{0} \bullet S^{0} \leq \varepsilon
$$

Taking logarithms on both sides, we have

$$
k \log (1-\theta)+\log \left(X^{0} \bullet S^{0}\right) \leq \log \varepsilon
$$

Applying inequality $\theta+\log (1-\theta) \leq 0(0 \leq \theta<1)$, we just need that the following inequality holds

$$
k \theta \geq \log \left(X^{0} \bullet S^{0}\right)-\log \varepsilon=\log \frac{X^{0} \bullet S^{0}}{\varepsilon} .
$$

This completes the proof of the lemma.

Theorem 4.9. Let $\theta=\frac{1}{2 \sqrt{n}}$, then the algorithm requires at most

$$
O\left(\sqrt{n} \log \frac{X^{0} \bullet S^{0}}{\varepsilon}\right)
$$

iterations.

Proof. Let $\theta=\frac{1}{2 \sqrt{n}}$, by using Lemma 4.8, the theorem is easily proof.

Corollary 4.10. If let $X^{0}=S^{0}=E$, the iteration bound is

$$
O\left(\sqrt{n} \log \frac{n}{\varepsilon}\right)
$$

which is the same as $L O$ and SDO and is also the currently best known iteration bound for the algorithm with small-update method. 


\section{Conclusions}

In this paper, we proposed a new primal-dual interior-point algorithm for CQSDO problem with full-Newton step. We used a new technique to find the search directions and applied new method to analyze the algorithm for CQSDO problem. Moreover, we obtained the currently best known iteration bound for small-update methods, namely, $O\left(\sqrt{n} \log \frac{n}{\varepsilon}\right)$, which is the same as LO and SDO case.

\section{REFERENCES}

[1] M. Achache, A new primal-dual path-following method for convex quadratic programming. Comput. Appl. Math. 25 (2006) 97-110.

[2] I. Adler and F. Alizadeh, Primal-dual interior point algorithms for convex quadratically constrained and semidefinite optimization problems. Technical Report RRR-111-95, Rutger Center for Operations Research, Brunswick, NJ (1995).

[3] A.Y. Alfakih, A. Khandani and H. Wolkowicz, Solving Euclidean distance matrix completion problems via semidefinite programming. Comp. Optim. Appl. 12 (1999) 13 C30.

[4] Y.Q. Bai and G.Q. Wang, A new primal-dual interior-point algorithm for second-order cone optimization based on kernel function. Acta Math. Sinica (English Series) 23 (2007) 20272042.

[5] Y.Q. Bai, C. Roos and M.El. Ghami, A comparative study of kernel functions for primal-dual interior-point algorithms in linear optimization. SIAM J. Optim. 15 (2004) 101-128.

[6] Z. Darvay, New interior-point algorithms in linear optimization. Adv. Model. Optim. 5 (2003) $51-92$.

[7] R.A. Horn and R.J. Charles, Topics in Matrix Analysis. Cambridge University Press, UK (1991).

[8] R.A. Horn and C.R. Johnson, Matrix analysis. Cambridge University Press (1990).

[9] E. de Klerk, Aspects of Semidefinite Programming: Interior Point Algorithms and Selected Applications. Kluwer Academic Publishers, Dordrecht, The Netherlands (2002).

[10] M. Kojima, M. Shida and S. Shindoh, Reduction of Monotone Linear Complemen-tarity Problems over Cones to Linear Programs over Cones. Acta Mathematica Vietnamica 22 (1997) 147-157.

[11] M. Kojima, S. Shindoh and S. Hara, Interior-point methods for the monotone linear complementarity problem in symmetric matrices. SIAM J. Optim. 7 (1997) 86-125.

[12] Y.E. Nesterov and M.J. Todd, Primal-dual interior-point methods for self-scaled cones. SIAM J. Optim. 8 (1998) 324-364.

[13] J.W. Nie and Y.X. Yuan, A Potential Reduction Algorithm for an Extended SDP. Science In China (Series A) 43 (2000) 35-46.

[14] J.W. Nie and Y.X. Yuan, A Predictor-Corrector Algorithm for QSDP Combining and Newton Centering Steps. Ann. Oper. Res. 103 (2001) 115-133.

[15] K.C. Toh, Inexact Primal-Dual Path-Following Algorithms for a Convex Quadratic SDP. Math. Program. 112 (2008) 221-254.

[16] K.C. Toh, R.H. Tütüncü and M.J. Todd, Inexact primal-dual path-following algorithms for a special class of convex quadratic SDP and related problems. Pac. J. Optim. 3 (2007) $135-164$.

[17] J. Peng, C. Roos and T. Terlaky, Self-regular functions and new search directions for linear and semidefinite optimization. Math. Program. 93 (2002) 129-171.

[18] G.Q. Wang and Y.Q. Bai, A new primal-dual path-following interior-point algorithm for semidefinite optimization. J. Math. Anal. Appl. 353 (2009) 339-349. 
[19] G.Q. Wang and Y.Q. Bai, Primal-dual interior point algorithm for convex quadratic semidefinite optimization. Nonlinear Anal. 71 (2009) 3389-3402.

[20] G.Q. Wang, Y.Q. Bai and C. Roos, Primal-dual interior-point algorithms for semidefinite optimization based on a simple kernel function. J. Math. Model. Algorithms 4 (2005) 409433.

[21] H. Wolkowicz, R. Saigal and L. Vandenberghe, Handbook of Semidefinite Programming, Theory, Algorithms, and Applications. Kluwer Academic Publishers, Dordrecht, The Netherlands (2000)

[22] Y. Zhang, On extending some primal-dual interior-point algorithms from linear programming to semidefinite programming. SIAM J. Optim. 8 (1998) 365-386. 